\title{
Effect of Nalbuphine on Hemdynamic Values and Bispectral Indices during Total Intravenous Anesthesia (TIVA) with Propofol and Remifentanil
}

\author{
Department of Anesthesiology and Pain Medicine, Chungnam National University College of Medicine, Daejeon, Korea
}

Woosuk Chung, M.D., Youngkwon Ko, M.D., Heesuk Yoon, M.D., and Jungun Lee, M.D.

Background: Remifentanil requires a long acting agent for postoperative pain control, and Nalbuphine, a long acting agonist-antagonist, causes less respiratory depression than pure $\mu$-agonists. However, Nalbuphine can also cause additional distress when used with a pure $\mu$-agonist. Therefore, we evaluated the effects of nalbuphine during TIVA with remifentanil and propofol.

Methods: 56 ASA class I, II adult patients undergoing minor surgery were included in this study. After maintaining BIS values between $40-60$ as well as a relatively similar blood pressure (BP) and heart rate (HR) for 20 minutes without changing the target concentrations of anesthesia during surgical procedures, the subjects received either $0.1 \mathrm{ml} / \mathrm{kg}$ of normal saline or nalbuphine intravenously. Hemodynamic and BIS variables were then recorded for 20 minutes, during which time the target concentrations were not modified. The BIS values, heart rate, and mean arterial pressure were then compared between groups using t-tests, with a $\mathrm{P}<0.05$ being considered statistically significant.

Results: The mean BIS and HR measured at each interval in the nalbuphine group were not significantly different from those of the control group. However, 10, 15 and 20 minutes after the administration of nalbuphine, the mean systolic BP and the mean arterial $\mathrm{BP}$ of the treatment and control groups were significantly different $(\mathrm{P}<0.05)$. In addition, the mean diastolic $\mathrm{BP}$ was also significantly different 10 and 20 minutes after the treatment was administered $(\mathrm{P}<0.05)$. Postoperative pain was well controlled and none of the patients reported intraoperative awareness.

Conclusions: Although nalbuphine seems to cause distress, which appeared as an increase in BP, it may still be used in combination with propofol and remifentanil because it did not cause a significant increase in the HR and BIS values. (Korean J Anesthesiol 2007; 53: $\mathrm{S}$ 7 11)

Key Words: BIS, hemodynamics, nalbuphine, propofol, remifentanil, TIVA.

\section{INTRODUCTION}

The development of intravenous drugs with more rapid onset and shorter recovery profiles (propofol, remifentanil), combined with technological advancements in infusion methods (target-controlled infusion, TCI), have made TIVA a widely used method of general anesthesia. ${ }^{1)}$

Remifentanil, a pure $\mu$ receptor agonist, is metabolized by blood and tissue estrases. Because its ultrashort action is due to metabolism rather than distrbution, it does not accumulate with

Received : June 4, 2007

Corresponding to : Youngkwon Ko, Department of Anesthesiology and Pain Medicine, College of Medicine, Chungnam National University, 640, Daesa-dong, Jung-gu, Daejeon 301-721, Korea. Tel: 82-42280-7841, Fax: 82-42-280-7968, E-mail: ane84@cnuh.co.kr repeated dosing or prolonged infusion making it ideal for TIVA. ${ }^{2)}$ The problem with remifentanil is that because of its ultrashort action, postoperative pain must be controlled with other long acting opioids. A long acting pure $\mu$ receptor agonist seems ideal, but pure $\mu$ agonists can cause complications such as respiratory depression, which can be dangerous in the recovery room. ${ }^{3)}$ On the other hand, nalbuphine which is an agonist-antagonist causes less respiratory depression by activating the supraspinal and spinal $\kappa$-receptors but has limited analgesia. ${ }^{2,4)}$ This makes nalbuphine quite useful in providing analgesia in mild to moderate postoperative pain. ${ }^{2)}$

The major down side is that nalbuphine, like other agonist-antagonist compounds, may interfere with the analgesia produced by pure $\mu$-receptor agonists such as remifentanil. ${ }^{4}$

In this study, we evaluated the effects of a nalbuphine bolus on BIS and hemodynamic variables during TIVA with propofol 
and remifentanil in minor surgeries. The purpose was to see if nalbuphine could safely provide postoperative pain relief without causing severe intraoperative distress which would appear as changes in hemodynamic variables and BIS values.

\section{MATERIALS AND METHODS}

After institutional and patient approval, 56 adult ASA class I, II patients, aged from 20 to 60 years were included in this prospective observational study. They were scheduled for minor surgical procedures including breast mass excision, mastectomy, lipoma excision, laparoscopic cholecystectomy, laparoscopic appendectomy and inguinal hernia repair. Exclusion criteria were central nervous system diseases, hemodynamic instability, ischemic heart diseases, renal or hepatic failure, or therapies with drugs that affect EEG activity.

The patients were randomly divided into two groups. The control group received saline $0.1 \mathrm{ml} / \mathrm{kg}$ and nalbuphine group recived $0.1 \mathrm{mg} / \mathrm{kg}$ intravenously. The two groups were similar in relation to age, sex, weight and height (Table 1). The two groups were also similar in the type and number of surgery (Table 2).

Premedication was done with an intramuscular injection of midazolam $2 \mathrm{mg}$ and glycoprryolate $0.2 \mathrm{mg}$. At the operation room, standard monitoring was applied (continous electrocardiography, non-invasive arterial blood pressure monitoring,

Table 1. Demographic Variables of Patients

\begin{tabular}{lcc}
\hline & Control group & Nalbuphine group \\
\hline Patients $\mathrm{n}$ & 28 & 28 \\
Gender $(\mathrm{M} / \mathrm{F})$ & $8 / 20$ & $7 / 21$ \\
Age $(\mathrm{yrs})$ & $47.5 \pm 8.1$ & $42.7 \pm 12.3$ \\
Weight $(\mathrm{kg})$ & $58.1 \pm 9.0$ & $59.8 \pm 8.1$ \\
Height $(\mathrm{cm})$ & $158.5 \pm 7.8$ & $161.0 \pm 9.9$ \\
\hline
\end{tabular}

All values expressed as means \pm S.D. or number of patients.

Table 2. Type and Number of Surgery

\begin{tabular}{lcc}
\hline & Control group & Nalbuphine group \\
\hline Breast mass excision & 5 & 7 \\
Mastectomy & 9 & 12 \\
Lipoma excision & 1 & 0 \\
Laparoscopic cholecystectomy & 8 & 6 \\
Laparoscopic appendectomy & 1 & 1 \\
Hernia (inguinal, femoral) repair & 4 & 2 \\
\hline
\end{tabular}

pulse oximetry and capnography). EEG activity was measured by means of a Bispectral Index monitor (BIS A-2000, software version 3.3, Aspect Medical Systems, USA). The BIS electrode (four-electrode sensor) was positioned on the patient's forehead as recommended by the manufacturer.

Induction was performed with a target-controlled infusion (TCI) of remifentanil followed by propofol (Orchestra infusion workstation, V03.OS-1, Fresenius Vial, France). The initial target of remifentanil was $3.0-4.0 \mathrm{ng} / \mathrm{ml}$ and propofol $3.0-4.0 \mu \mathrm{g} / \mathrm{ml}$ at effect site, respectively. Neuromuscular blockade was obtained with vecuronium or rocuronium. After tracheal tube insertion, ventilation was adjusted to maintain normocapnia (by end tidal $\mathrm{CO} 2$ monitoring), using $1.5 \mathrm{~L} / \mathrm{min} \mathrm{O} 2$ and $1.5 \mathrm{~L} /$ min medical air. Target concentrations were adjusted to keep the BIS value between 40-60 and relatively maintain similar blood pressure (BP) and heart rate (HR) compared to preoperative BP and HR.

During surgical procedures, we adjusted the target concentration of propofol and remifentanil until BIS, HR and BP were stabilized for over 20 minutes without changing the target concentration of propofol and remifentanil. The patients then received either saline $0.1 \mathrm{ml} / \mathrm{kg}$ (control group) or nalbuphine 0.1 $\mathrm{mg} / \mathrm{kg}$ (nalbuphine group) intravenously. All hemodynamic and BIS variables were recorded at the following times: before administration ( 0 minute), and at 5, 10, 15, and 20 minute after administration. The target concentrations of propofol and remifentanil were not modified during this period. The patients position was also unchanged. At the recovery room, intraoperative awareness was evaluated simply by asking if the patient remembered anything during the operation before leaving the recovery room after full recovery.

Demographic data were expressed as mean \pm SD. The BIS values, heart rate, and mean arterial pressure were compared between groups using t-tests. $\mathrm{P}<0.05$ was considered statistically significant.

\section{RESULTS}

Nalbuphine groups' BIS values measured at each interval were not significantly different from the control group $(\mathrm{P}>0.05$, Fig. 1). HR measured at each interval also showed the same results $(\mathrm{P}>0.05$, Fig. 2). But there were significant differences in the systolic blood pressures measured at 10, 15 and $20 \mathrm{mi}-$ nute after nalbuphine injection when compared to the control group $(\mathrm{P}<0.05$, Fig. 3). Mean arterial blood pressures also showed the same results $(\mathrm{P}<0.05$, Fig. 4). There were sig- 


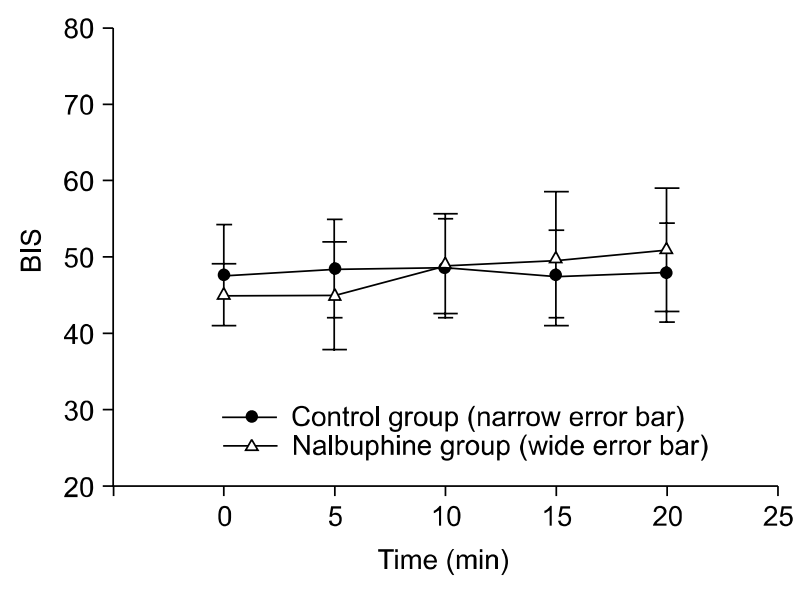

Fig. 1. This figure shows the changes in BIS values after injection of nalbuphine and normal saline. Each point on the graph represents the mean \pm SEM. There is no significant difference between groups.

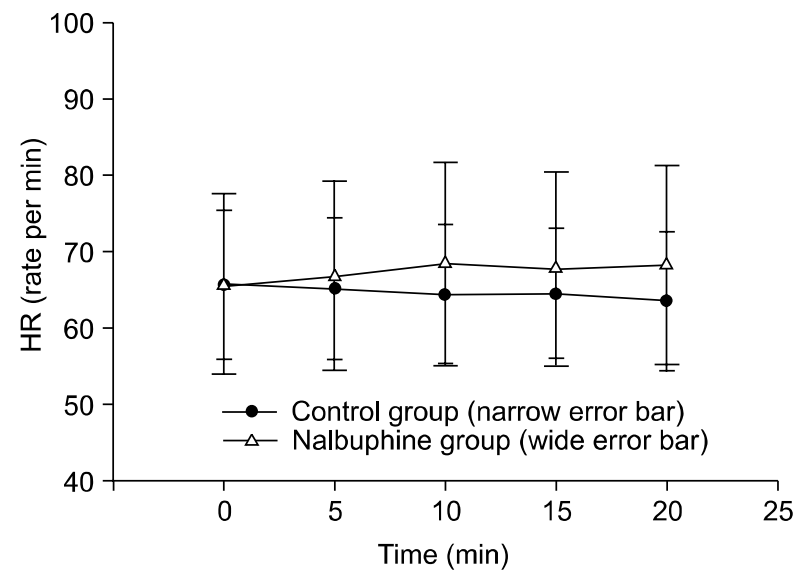

Fig. 2. This figure shows the changes in heart rate (HR) after injection of nalbuphine or saline. Each point on the graph represents the mean \pm SEM. There is no significant difference between groups.

nificant differences in the diastolic blood pressures measured at 10, 20 minute after nalbuphine injection $(\mathrm{P}<0.05$, Fig. 5). 3 patients in the nalbuphine group complained of intolerable postoperative pain at the recovery room. However pain was well controlled after an additional intravenous administration of $5 \mathrm{mg}$ of nalbuphine. When questioned, none of the patients complained of intraoperative awareness.

\section{DISCUSSION}

Remifentanil, a fentanyl derivative, is an ultra-short acting,

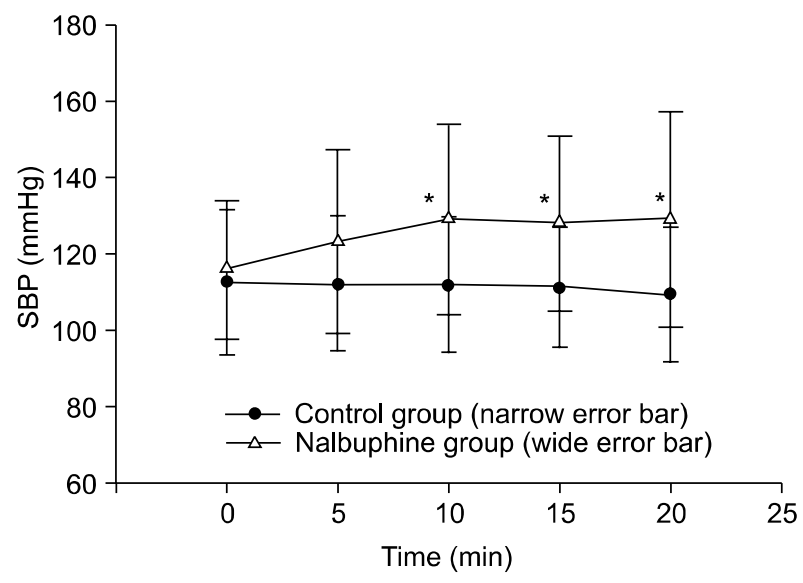

Fig. 3. This figure shows the changes in systolic blood pressure (SBP) after injection of nalbuphine or saline. Each point on the graph represents the mean \pm SEM. SBP increases in the nalbuphine group and is significantly different from the control group at 10, 15 and 20 minutes after injection $(\mathrm{P}<0.05)$. *: $\mathrm{P}<0.05$, compared with the control group.

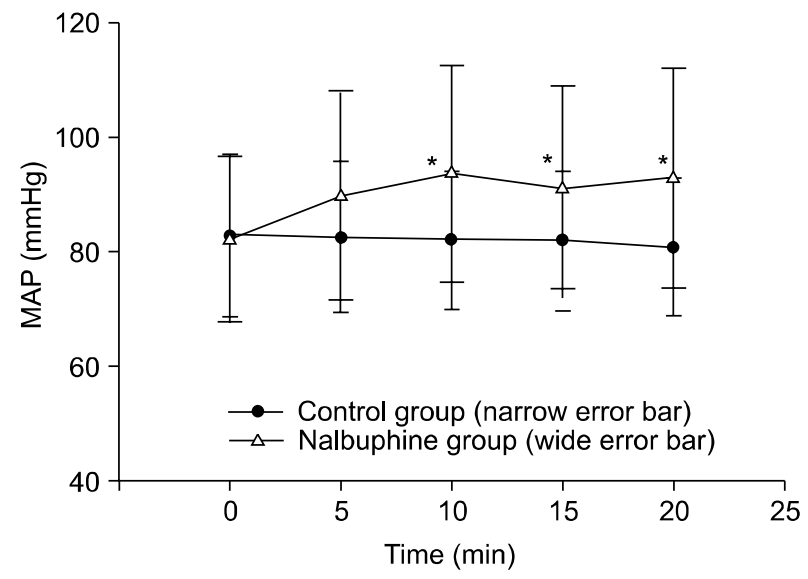

Fig. 4. This figure shows the changes in mean arterial blood pressure (MAP) after injection of nalbuphine or saline. Each point on the graph represents the mean \pm SEM. MAP increases in the nalbuphine group and is significantly different from the control group at 10,15 and 20 minutes after injection $(\mathrm{P}<0.05)$. *: $\mathrm{P}<$ 0.05 , compared with the control group.

nonspecific esterase-metabolised, selective $\mu$-opioid receptor agonist with a pharmocodynamic profile typical of opioid analgesic agents. ${ }^{5)}$ Irrespective of the duration of the infusion, the rapid offset of action and short context-sensitive half-time makes this drug a valuable opioid analgesic option for use during general anesthesia where rapid, titratable, intense analgesia of variable duration, and a fast and predictable recovery are required. ${ }^{5)}$ But because of this rapid offset of action and short context-sensitive 


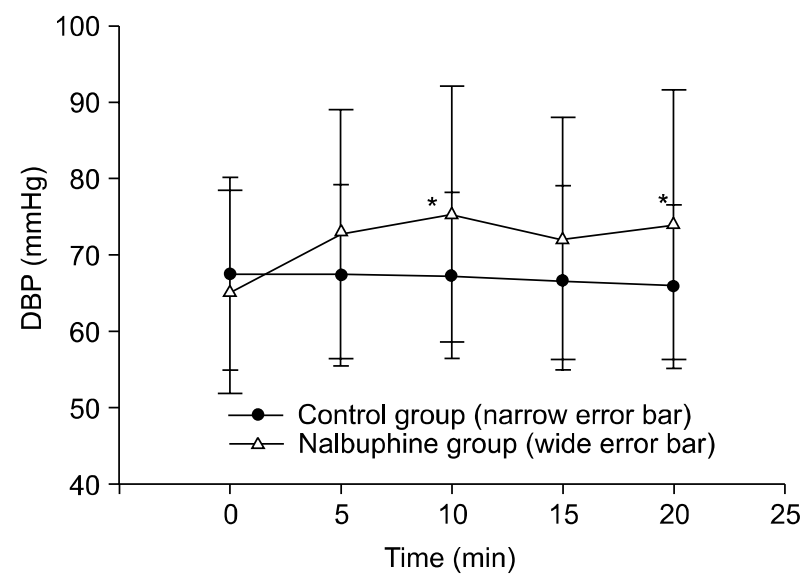

Fig. 5. This figure shows the changes in diastolic blood pressure (DBP) after injection of nalbuphine or saline. Each point on the graph represents the mean \pm SEM. DBP increases in the nalbuphine group and is significantly different from the control group at 10 and 20 minutes after injection $(\mathrm{P}<0.05)$. DBP is not significantly different between the two groups at 15 minutes after administration $(\mathrm{P}>0.05)$. *: $\mathrm{P}<0.05$, compared with control group.

half-time, a long acting postoperative pain control agent must be used. Although a long acting pure $\mu$ agonist seems most proper they may cause life threatening complications, such as respiratory depression. ${ }^{3)}$

Nalbuphine is an agonist-antagonist opioid that is structurally related to oxymorphone and naloxone. Nalbuphine binds to $\mu$ -receptors as well as $\kappa$ - and $\delta$-receptors. Although activation of supraspinal and spinal $\kappa$-receptors results in limited analgesia, it also limits respiratory depression and sedation, making it quite useful in surgeries with mild to moderate pain. ${ }^{4)}$ The problem with using nalbuphine, just like any other agonist-antagonist compounds, is that it can interfere with the analgesia produced by the pure $\mu$-receptor agonist remifentanil. ${ }^{4}$

In order to see if nalbuphine could used to control postoperative pain during TIVA with propofol and remifentanil, traditional methods as well as BIS were used in order to analyze the effects of nalbuphine. ${ }^{\text {) }}$

Traditionally, the effects of anesthetic drugs were measured by observing changes in HR, blood pressure, breathing pattern, and the presence of movement. Although these traditional methods are still useful, they can not be a direct indicator of the sedative and hypnotic effects of anesthesia. Electroencephalography (EEG) is more appropriate and noninvasive. Continous monitoring has recently become possible with the invention of bispectral analysis (BIS). ${ }^{6}$ Explicit recall, otherwise awareness, was evident in ap- proximately $0.2 \%$ of cases when no neurophysiological monitoring was used to guide the conduction of anesthesia using muscle relaxants. The use of BIS monitoring during general anesthesia was associated with the significantly reduced incidence of awareness. $^{7}$

In this study, nalbuphine was administered during surgical procedures, after confirming that BIS was kept between 40-60 and $\mathrm{HR}$ and BP were maintained similar to the preoperative BP and HR for over 20 minutes at the same concentration of propofol and remifentanil during intravenous anesthesia. The results were a non-significant increase in BIS value and HR at each interval. But there were significant increases in systolic, diastolic, mean arterial blood pressure $(\mathrm{P}<0.05)$.

BIS values above 60 were shown in 4 patients who received nalbuphine. 1 patient showed a value of 62 after 20 minute of injection, another patient showed a value of 63 after 5 and 20 minute of injection. The other 2 patients showed BIS values above 60 after 15 and 20 minute of injection. 61, 64 in one patient and 66, 67 in the other. None of the patients had a prolonged period ( $>5$ minute) of a high BIS value (BIS $>60$ ). Although BIS values between 45 and 60 are recommended for anesthetic maintenance during general anesthesia, loss of consciousness is correlated to BIS values between 68 and $75^{8)}$ Another study showed that explicit recall is evident in only a fraction of patients showing BIS values $>60$ for 4 minute or more. This may be due to due to amnesia but also to interindividual variability in the relation between cognitive capacity and BIS. ${ }^{7}$

Considering these results, nalbuphine seems to interfere with the analgesia produced by remifentanil by showing an increase in blood pressure. Even with this interference of analgesia there were no significant increase mean BIS values and none of the patients reported intraoperative awareness after full recovery at the recovery room. Also only 3 patients in the nalbuphine group complained of intolerable pain in the recovery room, but after an additional intravenous administration of $5 \mathrm{mg}$ nalbuphine the pain became tolerable. This study suggests that evan though nalbuphine interferes with the analgesia produced by remifentanil appearing as an increase in blood pressure it may still be used for minor operations in healthy patients due to the fact that it did not cause a significant increase in HR and BIS values.

\section{REFERENCES}

1. White PF, Romero G: Nonopioid intravenous anesthesia. In: 
Clinical Anesthesia. 5th ed. Edited by Barash PG, Cullen BF, Stoelting RK: Philadelphia, Lippincott Williams \& Wilkins. 2006, pp 347.

2. Coda BA: Opioids. In: Clinical Anesthesia. 5th ed. Edited by Barash PG, Cullen BF, Stoelting RK: Philadelphia, Lippincott Williams \& Wilkins. 2006, pp 371-7.

3. Fukuda K: Intravenous opioid anesthesia. In: Miller's Anesthesia. 6th ed. Edited by Miller RD: Philadelphia, Elsevier Inc. 2005, pp 392-4.

4. Fukuda K: Intravenous opioid anesthesia. In: Miller's Anesthesia. 6th ed. Edited by Miller RD: Philadelphia, Elsevier Inc. 2005, pp
419-20.

5. Scott LJ, Perry CM: Spotlight on remifentanil for general anaesthesia. CNS Drugs 2005; 19: 1069-74.

6. Rampil IJ: A primer for EEG signal processing in anesthesia. Anesthesiology 1998; 89: 980-1002.

7. Ekman A, Lindholm ML, Lennmarken C, Sandin R: Reduction in the incidence of awareness using BIS monitoring. Acta Anaesthesiol Scand 2004; 48: 20-6.

8. Johansen JW: Update on bispectral index monitoring. Best Pract Clin Anaesthesiol 2006;20:81-99. 